\title{
High yield of culture-based diagnosis in a TB-endemic setting
}

\author{
Anne-Marie Demers ${ }^{1,2}$, Suzanne Verver ${ }^{3,4}$, Andrew Boulle ${ }^{5}$, Robin Warren ${ }^{6}$, Paul van Helden ${ }^{6}$, Marcel A Behr ${ }^{7,8^{*}}$ \\ and David Coetzee ${ }^{5}$
}

\begin{abstract}
Background: In most of the world, microbiologic diagnosis of tuberculosis (TB) is limited to microscopy. Recent guidelines recommend culture-based diagnosis where feasible.

Methods: In order to evaluate the relative and absolute incremental diagnostic yield of culture-based diagnosis in a high-incidence community in Cape Town, South Africa, subjects evaluated for suspected TB had their samples processed for microscopy and culture over a 21 month period.

Results: For 2537 suspect episodes with 2 smears and 2 cultures done, 20.0\% (508) had at least one positive smear and 29.9\% (760) had at least one positive culture. One culture yielded 1.8 times more cases as 1 smear (relative yield), or an increase of $12.0 \%$ (absolute yield). Based on the latter value, the number of cultures needed to diagnose (NND) one extra case of TB was 8, compared to 19 if second specimens were submitted for microscopy.

Conclusion: In a high-burden setting, the introduction of culture can markedly increase TB diagnosis over microscopy. The concept of number needed to diagnose can help in comparing incremental yield of diagnosis methods. Although new promising diagnostic molecular methods are being implemented, TB culture is still the gold standard.
\end{abstract}

Keywords: Tuberculosis, Diagnosis, Culture, Microscopy

\section{Background}

For years, the World Health Organization (WHO) and the International Union against Tuberculosis and Lung Disease (IUATLD) recommended sputum smear microscopy as the cornerstone to diagnose pulmonary $\mathrm{TB}$, as smearpositive subjects are most contagious [1,2]. However, since smear microscopy has a detection limit of about 5000-10 000 organisms/mL [3] - compared to 10-100 organisms/ $\mathrm{mL}$ for culture [4] - specimens with $<5000$ bacteria are negative by microscopy.

In settings where microscopy and culture are both done, such as developed countries, approximately half of culture positive specimens have negative smears [5-8]. Based on this, one anticipates a doubling of case detection if culture methods are introduced into a setting that only does microscopy.

\footnotetext{
* Correspondence: marcel.behr@mcgill.ca

${ }^{7}$ Department of Medicine, McGill University, Montreal, Québec, Canada

${ }^{8}$ McGill University Health Centre, Room A5.156, 1650 Cedar Avenue,

Montreal, QC H3G 1A4, Canada

Full list of author information is available at the end of the article
}

WHO recommends the use of liquid culture in low income settings where feasible [9]. However, other authorities consider the role of culture to be primarily for surveillance and diagnosis of MDR-TB [10]. Little is known on the yield of culture in the context of national TB control programmes in high incidence countries. We have evaluated the relative (RY) and absolute yield (AY) of introducing culture-based diagnosis in a high incidence setting (Cape Town, South Africa).

\section{Methods \\ Study design \\ Prospective cohort study in regular programme setting}

Study setting This study was conducted in Gugulethu, a residential area in Cape Town, South Africa, as part of a molecular epidemiology study. At the time of the study, the notified incidence of TB was 992/100 000 and the antenatal HIV prevalence was $28.1 \%$ [11]. The setting is still similar now with respect to incidence rate and proportion of patients HIV infected [12].

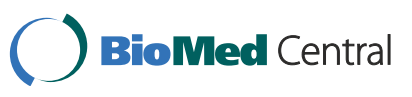

(C) 2012 Demers et al.; licensee BioMed Central Ltd. This is an Open Access article distributed under the terms of the Creative Commons Attribution License (http://creativecommons.org/licenses/by/2.0), which permits unrestricted use, distribution, and reproduction in any medium, provided the original work is properly cited. 


\section{Inclusion criteria}

From 1 April 2002 to 31 December 2003, subjects older than 14 years presenting to the NY1 Gugulethu TB clinic with signs or symptoms of pulmonary TB were considered suspects and included in the study. Because some individuals presented more than once during the study period, we analyzed the data by suspect episodes (SE) and not patients. We defined a new SE if the same person returned to the clinic more than 6 weeks after a previous evaluation. A previous analysis describing a method to assess laboratory cross-contamination has already been published [13].

\section{Data collection}

Routine diagnosis and treatment procedures were followed by clinical staff, except that culture was added to the smear-based diagnosis recommended by the South African TB Guidelines, which restrict cultures to retreatment cases [14]. Every effort was made to collect 2 sputum samples for each SE. Additional smears and cultures were done if judged necessary by the clinic staff. Chest $\mathrm{x}$-rays were not done routinely as per national guidelines.

Demographic and laboratory data were collected for all SE. No supplementary procedures were performed on patients. For treatment episodes, outcome information was obtained from the clinical notes, patient interviews and the TB register. Patients started on TB treatment were interviewed by the study team after informed consent was obtained. Since some patients might have been treated at another TB clinic in the area, we manually matched by name and demographic data the untreated positive culture SE with the subdistrict electronic TB register for July 2002 to Oct 2005.

HIV voluntary counseling and testing was offered to every patient put on TB treatment. HIV information was only available for those started on treatment and was collected from patient interviews and record reviews. At the time of the study, antiretrovirals were not routinely available for HIV positive patients. The protocol was approved by the research ethics committee of the Faculty of Health Sciences, University of Cape Town (REC REF 048/2001).

\section{Laboratory procedures}

Sputum specimens were collected at the Gugulethu clinic and kept refrigerated until transported later the same day to the National Health Laboratory Services (NHLS) in Cape Town where they were processed for fluorescence (auramine) microscopy and culture. Smear results were reported as per WHO/IUATLD classification [15]. Cultures were done both on one Löwenstein-Jensen and one BACTEC MGIT $960{ }^{\circledR}$ bottle (BD Diagnostic
Systems, Sparks, MD) and incubated for 8 weeks. If either culture method was positive, the sample was reported positive. Unfortunately, the results for solid and liquid media were not recorded individually. In addition to biochemical methods, positive cultures were speciated as $M$. tuberculosis complex using the Accuprobe ${ }^{\circledR}$ (Gen-Probe Inc., San Diego, CA). Susceptibility testing for isoniazid (INH) and rifampicin (RIF) was done using the indirect proportion method. Details on the laboratory procedure have been described elsewhere [13].

\section{Definitions}

Only diagnosis specimens were analysed. We considered scanty smears to be positive [16-18]. SE with culture results other than negative or positive for $M$. tuberculosis (i.e. non-tuberculous mycobacteria (NTM), lost viability or contaminated) were excluded from the analysis as it was not possible to assume with certainty that they were negative or positive. For each SE, both smear and culture results were ordered by timing of specimen collection and classified according to the following patterns of successive specimens: $\mathrm{N}$ ( $\mathrm{N}=$ negative), $\mathrm{P}$ ( $\mathrm{P}=$ positive), NN, NP, PP, PN, NNN, NNP, NPN, NPP, etc. Smear and culture results were then combined together per SE. A SE was classified as smear positive if any of the smears was positive. A SE was classified as culture positive if any of the cultures was positive for $M$. tuberculosis complex. Smear and culture results were then grouped together, defining SE as SNCP (smear negative culture positive), SPCP (smear positive culture positive), SPCN (smear positive culture negative) or SNCN (smear negative culture negative).

Because our study was done under routine conditions, not all TB suspects had 2 specimens collected. In order to correctly calculate the incremental yields, we added the observed proportions of positive smears or cultures to the expected proportion for the missing specimens, using a method described by Rieder et al. (Appendix) [19]. Since less than $10 \%$ of SE had a 3rd smear or culture done, we could not calculate accurately the yields for the third specimen. We therefore restricted the

Table 1 Definitions

\begin{tabular}{lll}
\hline Definition & $=$ & Formula \\
\hline $\begin{array}{l}\text { Relative incremental yield of positive per method 2 } \\
\text { method* 1 vs method 2 (RY) }\end{array}$ & $=$ & $\begin{array}{l}\% \text { positive per method } \\
\text { Absolute incremental yield of } \\
\text { method 2 vs method 1 (AY) }\end{array}$ \\
$\begin{array}{ll}\text { Number needed to diagnose one } \\
\text { extra case of TB (NND) }\end{array}$ & $=$ & $\begin{array}{l}1 \\
\text { method } 1\end{array}$ \\
\hline
\end{tabular}

Legend: *Method: can either be 1 smear, 2 smears, 1 culture, 2 cultures. 


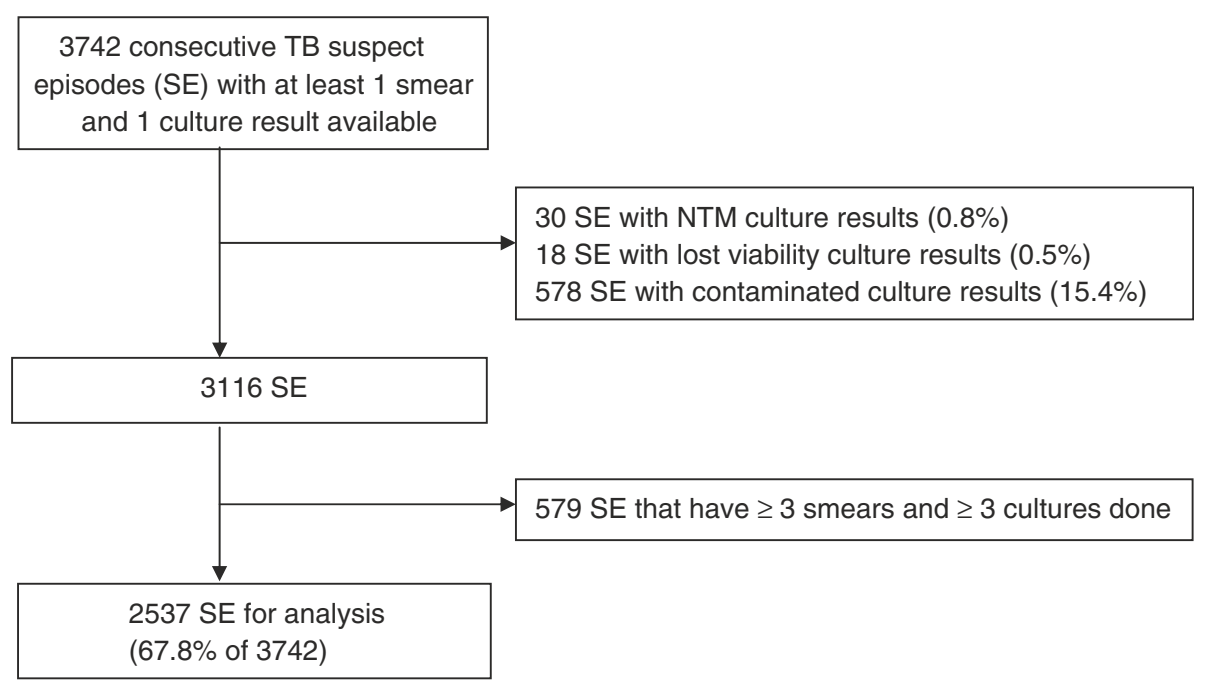

Figure 1 Study flow diagram. Legend. SE = Suspect Episodes, NTM = Non-tuberculous mycobacteria.

analysis to SE with $\leq 2$ smears \& $\leq 2$ cultures. We also regrouped our initial patterns of successive specimens into Rieder's patterns, adapted for 2 specimens: N9 (where $\mathrm{N}$ is negative and 9 is a missing specimen), $\mathrm{NN}$, $\mathrm{NP}$ and $\mathrm{Px}$ (where $\mathrm{P}$ is positive and $\mathrm{x}$ is a subsequent result of no interest for the calculation of incremental yields) (Appendix)[19].To calculate the relative and absolute yield of smears and cultures, we used the formulas shown in Table 1. We defined the number needed to diagnose (NND) as the reciprocal of the absolute yield. We did not use another proposed definition for NND [20] because it did not consider prevalence [21].

\section{Data analysis}

Data were collected and analyzed using Microsoft Access 2002 database and Stata software, version 10 (Stata Corporation). The chi-squared test was used to compare proportions.

\section{Results}

During the study period, 3742 SE had at least 1 smear and 1 culture done. After excluding the SE which were contaminated, showed the presence of a NTM or lost viability, and after removing the $\mathrm{SE}$ with $\geq 3$ smears or cultures, 2537 SE were left for analysis $(67.8 \%$ of 3742$)$ (Figure 1). These 2537 SE represented 2207 individuals: $62 \%$ were male with a median age of 35 years (interquartile range 27-45). Of note, only 1 of the 30 excluded SE with NTM had a mixed infection with M. tuberculosis.

\section{Smears and cultures results}

In Table 2, smear and culture results are presented for the first and for both specimens (combined field). The sensitivity of smear compared to culture was $50.6 \%$ and $58.9 \%$ respectively. Of the $2537 \mathrm{SE}, 20.0 \%$ had at least one positive smear and $29.9 \%$ had at least one positive culture after 2 specimens (expected proportion of positives in Table 3). Using all smear positive SE as a denominator, the fraction of SE positive on the first smear (Fd1) was $74.3 \%$ and the incremental yield (IY) of the second smear (Fd2) was 25.7\% (Table 3). For cultures, $89.8 \%$ of positive cultures were positive on the first specimen, and the IY of the second culture was $10.2 \%$. The number of specimens needed to find

Table 2 Smear and culture results per SE for the $1^{\text {st }}$ specimen and for both specimens

\begin{tabular}{|c|c|c|c|c|c|c|c|c|c|}
\hline \multicolumn{2}{|c|}{ 1st specimen } & \multicolumn{3}{|l|}{ Culture } & \multicolumn{2}{|l|}{ After 2 specimens } & \multicolumn{3}{|c|}{ Culture (combined) } \\
\hline Smear & $P$ & $\begin{array}{l}\mathbf{P} \\
345\end{array}$ & $\frac{\mathbf{N}}{32}$ & $\begin{array}{l}\text { Total } \\
377\end{array}$ & Smear (combined) & $P$ & $\begin{array}{l}\mathbf{P} \\
432\end{array}$ & $\begin{array}{l}\text { N } \\
45\end{array}$ & $\begin{array}{r}\text { Total } \\
477\end{array}$ \\
\hline & $N$ & 337 & 1823 & 2160 & & $\mathrm{~N}$ & 301 & 1759 & 2060 \\
\hline & Total & 682 & 1855 & 2537 & & Total & 733 & 1804 & 2537 \\
\hline & $\mathrm{Se}=$ & $\mathrm{SPCP} / \mathrm{CP}$ & $50.6 \%$ & & & $\mathrm{Se}=$ & $\mathrm{SPCP} / \mathrm{CP}$ & $58.9 \%$ & \\
\hline & $S p=$ & $\mathrm{SNCN} / \mathrm{CN}$ & $98.3 \%$ & & & $S p=$ & $\mathrm{SNCN} / \mathrm{CN}$ & $97.5 \%$ & \\
\hline & $P P V=$ & $\mathrm{SPCP} / \mathrm{SP}$ & $91.5 \%$ & & & $P P V=$ & $\mathrm{SPCP} / \mathrm{SP}$ & $90.6 \%$ & \\
\hline & $N P V=$ & $\mathrm{SNCN} / \mathrm{SN}$ & $84.4 \%$ & & & $N P V=$ & $\mathrm{SNCN} / \mathrm{SN}$ & $85.4 \%$ & \\
\hline
\end{tabular}

Legend: $\mathrm{S}=$ smear, $\mathrm{C}=$ culture, $\mathrm{P}=$ positive, $\mathrm{N}=$ negative, $\mathrm{Se}=$ Sensitivity, $\mathrm{Sp}=$ Specificity, $\mathrm{PPV}=$ Positive predictive value, $\mathrm{NPV}=$ Negative predictive value. 
Table 3 Smear and culture results for all SE, smear negative and smear positive SE

\begin{tabular}{|c|c|c|c|c|}
\hline Description & All smears & All cultures & Cultures in SN SE & Cultures in SP SE \\
\hline Number of SE & 2537 & 2537 & 2060 & 477 \\
\hline Observed \# of P SE after 1 specimen & 377 & 682 & 253 & 429 \\
\hline Observed \# of P SE after 2 specimens & 477 & 733 & 301 & 432 \\
\hline Proportion of P SE on $1^{\text {st }}$ specimen & 0.149 & 0.269 & 0.123 & 0.899 \\
\hline Proportion of NP SE & 0.060 & 0.042 & 0.040 & 0.103 \\
\hline \# of P SE missed by failing to do $2^{\text {nd }}$ specimen & 31 & 27 & 25 & 2 \\
\hline Expected \# of P SE after 1 specimen & 377 & 682 & 253 & 429 \\
\hline Expected \# of P SE after 2 specimens & 508 & 760 & 326 & 434 \\
\hline Expected proportion of P SE after 1 specimen & 0.149 & 0.269 & 0.123 & 0.899 \\
\hline Expected proportion of P SE after 2 specimens & 0.200 & 0.299 & 0.158 & 0.910 \\
\hline Potential IY for $1^{\text {st }}$ specimen & 0.743 & 0.898 & 0.776 & 0.989 \\
\hline Potential IY for $2^{\text {nd }}$ specimen & 0.257 & 0.102 & 0.224 & 0.011 \\
\hline Overall fraction of $\mathrm{P}$ on $1^{\text {st }}$ specimen & 0.149 & 0.269 & 0.123 & 0.899 \\
\hline Overall fraction of $\mathrm{P}$ on $2^{\text {nd }}$ specimen & 0.052 & 0.031 & 0.035 & 0.010 \\
\hline NND on $1^{\text {st }}$ specimen & 7 & 4 & 8 & 1 \\
\hline NND on $2^{\text {nd }}$ specimen & 19 & 33 & 28 & 96 \\
\hline
\end{tabular}

Legend: $\mathrm{S}=$ smear, $\mathrm{P}=$ positive, $\mathrm{N}=$ negative, $\mathrm{SE}=$ Suspect Episodes, NND = number needed to diagnose (see Methods for details), IY = incremental yield.

one case on the first smear was 7. When comparing the second smear to the first smear, 19 specimens would need to be examined before finding another positive one. For cultures, only 4 specimens would be needed to find a positive result on the first specimen but when comparing the yield of the second culture to the first culture, the NND would be 33 . Table 4 shows the results presented as RY, AY and NND to compare different screening strategies. The relative yield of doing 1 culture was about twice the yield of 1 smear (1.8), for an absolute yield of $12.0 \%$, meaning that in this setting, one can detect one additional case of TB

Table 4 Relative and absolute incremental yields and NND for smear and cultures

\begin{tabular}{|c|c|c|c|c|}
\hline \multirow[b]{2}{*}{ Yield } & & \multicolumn{3}{|c|}{ Relative incremental yield vs. } \\
\hline & & 1 st $\mathrm{S}$ & $2 \mathrm{~S}$ total & 1 st $C$ \\
\hline \multicolumn{5}{|l|}{ Expected \# of P after $1 \mathrm{~S}=377 / 2537=14.9 \%$} \\
\hline \multicolumn{2}{|l|}{ Expected \# of P after $2 S=508 / 2537=20.0 \%$} & \multicolumn{2}{|l|}{1.3} & \\
\hline \multicolumn{2}{|l|}{ Expected \# of P after $1 \mathrm{C}=682 / 2537=26.9 \%$} & 1.8 & 1.3 & \\
\hline \multicolumn{2}{|l|}{ Expected \# of P after 2 C $=760 / 2537=29.9 \%$} & 2.0 & 1.5 & 1.1 \\
\hline & & \multicolumn{2}{|c|}{ Absolute incremental yield vs. } & \\
\hline \multicolumn{2}{|l|}{ Yield } & 1st S & $2 \mathrm{~S}$ total & 1st C \\
\hline \multicolumn{5}{|l|}{ Expected \# of P after $1 \mathrm{~S}=377 / 2537=14.9 \%$} \\
\hline \multicolumn{2}{|l|}{ Expected \# of P after $2 S=508 / 2537=20.0 \%$} & $5.2 \%$ & & \\
\hline \multicolumn{2}{|l|}{ Expected \# of P after 1 C = 682/2537 = 26.9\% } & $12.0 \%$ & $6.9 \%$ & \\
\hline \multirow{2}{*}{\multicolumn{2}{|c|}{ Expected \# of P after $2 \mathrm{C}=760 / 2537=29.9 \%$}} & $15.1 \%$ & $9.9 \%$ & $3.1 \%$ \\
\hline & & NND vs. & & \\
\hline & NND & 1st $S$ & $2 \mathrm{~S}$ total & 1st C \\
\hline 1st Smear & 7 & & & \\
\hline 2 Smears total & 5 & 19 & & \\
\hline 1st Culture & 4 & 8 & 15 & \\
\hline 2 Cultures total & 3 & 7 & 10 & 33 \\
\hline
\end{tabular}

Legend: $\mathrm{S}=$ smear, $\mathrm{P}=$ positive, $\mathrm{N}=$ negative, $\mathrm{SE}=$ Suspect Episodes, NND = number needed to diagnose (see Methods for details), IY = incremental yield. Note: Expected \# of $\mathrm{P}$ after $1 \mathrm{~S}$ or $\mathrm{C}$ is same as Observed (see Appendix for details). 
by performing 8 additional cultures $(\mathrm{NND}=8)$. In contrast, one additional case was diagnosed when 19 patients submitted a second sample for smear microscopy (Tables 3 and 4).

We compared the culture results of the smear positive SE to the smear negative SE (Table 3). Smear positive specimens are expected to be culture positive so yields are presented mainly as a comparison to the smear negative SE. Although the potential IY for the first culture was lower at $77.6 \%$ compared to all cultures (89.8\%), it is still very high. The IY for a second culture in smear negative episodes was $22.4 \%$. Of the 733 culture positive SE, susceptibility results were available for 639 (87.2\%). Of these, only $7(1.1 \%)$ were resistant to isoniazid and rifampicin, precluding further analysis due to small numbers.

\section{HIV results}

The HIV status and outcome were available only for treated patients that had consented to HIV testing: this represented $345(13.6 \%)$ of the $2537 \mathrm{SE}$ and $302(41.2 \%)$ of the 733 culture confirmed SE. More than half of these 345 SE were HIV infected $(192 / 345=55.7 \%)$. The yield of the first smear was $38.5 \%$ in HIV positive patients (74/192) compared to $51.0 \%(78 / 153)$ in HIV negative patients (chi-square $=5.34, \mathrm{p}=0.021$ ). For HIV infected patients, the relative yield of 1 culture compared to 1 smear was higher than in HIV negative patients, with a relative yield of 2.1, an absolute yield of $43.2 \%$ and a NND of 2.3 (data not shown). However, these results should be interpreted with caution since the HIV status was known for only a small proportion of TB suspects.

\section{Discussion}

When culture was added to the routine smear-based diagnosis of TB suspects in a high incidence area of South Africa, we found that $29.9 \%$ of 2537 SE were culture positive, compared to $20.0 \%$ for smear microscopy. The relative yield of culture-based diagnosis was almost double, consistent with the observation that microscopy typically has a sensitivity of about $50 \%$ when

Table 5 Yields and NND calculated on some other recent studies that have used culture based diagnosis in TB suspects

\begin{tabular}{|c|c|c|c|c|c|}
\hline & Our study & Our study SN SE & Monkongdee et al. [32] & Boehme et al. [36] & Ssengooba et al. [35] \\
\hline Population & $\overline{\text { HIV pos and neg }}$ & $\overline{\text { SN in HIV pos and neg }}$ & All HIV pos & HIV pos and neg & $\overline{\text { All HIV pos and SN }}$ \\
\hline Pos on 1st S & 377 & & 36 & 561 & \\
\hline Pos on 25 & 508 & & 45 & & \\
\hline Pos on 1st C & 682 & 253 & 97 & 732 & 52 \\
\hline Pos on $2 \mathrm{C}$ & 760 & 326 & 113 & & 60 \\
\hline TB suspects tested & 2537 & 2060 & 1060 & 1462 & 170 \\
\hline$\%$ Pos on 1 st $\mathrm{S}$ & 0.149 & & 0.034 & 0.384 & \\
\hline$\%$ Pos on 25 & 0.200 & & 0.042 & & \\
\hline$\%$ Pos on 1 st C & 0.269 & 0.123 & 0.092 & 0.501 & 0.306 \\
\hline$\%$ Pos on $2 \mathrm{C}$ & 0.299 & 0.158 & 0.107 & & 0.353 \\
\hline RY $2 S$ vs $1 S$ & 1.3 & & 1.3 & & \\
\hline RY $1 C$ vs $1 S$ & 1.8 & & 2.7 & 1.3 & \\
\hline RY $1 C$ vs $2 S$ & 1.3 & & 2.2 & & \\
\hline RY $2 C$ vs $1 C$ & 1.1 & 1.3 & 1.2 & & 1.2 \\
\hline AY $2 S$ vs $1 S$ & 0.052 & & 0.008 & & \\
\hline AY $1 C$ vs $1 S$ & 0.120 & & 0.058 & 0.117 & \\
\hline AY $1 C$ vs $2 S$ & 0.069 & & 0.049 & & \\
\hline AY $2 C$ vs $1 C$ & 0.031 & 0.035 & 0.015 & & 0.047 \\
\hline NND $2 S$ vs 15 & 19 & & 118 & & \\
\hline NND $1 \mathrm{C}$ vs $1 \mathrm{~S}$ & 8 & & 17 & 9 & \\
\hline NND $1 C$ vs $2 S$ & 15 & & 20 & & \\
\hline NND $2 C$ vs $1 C$ & 33 & 28 & 66 & & 21 \\
\hline NND of 1st $S$ & 7 & & 29 & 3 & \\
\hline NND of $1 \mathrm{st} C$ & 4 & 8 & 11 & 2 & 3 \\
\hline
\end{tabular}

Legend: $\mathrm{S}=$ smear, $\mathrm{C}=$ culture, Pos = positive, Neg = negative, $\mathrm{RY}=$ Relative yield, $\mathrm{AY}=$ Absolute yield, $\mathrm{SE}=$ suspect episode, $\mathrm{NND}=$ number needed to diagnose. In the study by Monkongdee et al. [32], yields were reported using all culture positive cases as the denominator. The results shown here use all suspects tested as the denominator. 
compared to culture [5-8]. In this high TB setting, one can obtain an additional TB diagnosis with just 8 cultures, or alternatively by submitting 19 second specimens for microscopy.

Although smear is less sensitive than culture, nevertheless it has excellent PPV in high incidence areas [3]. Our data indicate that while many patients can be detected by microscopy in a high-prevalence setting [22], the addition of culture-based diagnosis can detect many more. Moreover, the present study used the more sensitive fluorescent technique to define a microscopypositive sample; the incremental yield of culture should be even greater in settings that do only light microscopy. Reviews on the sensitivity of smear compared to culture are based mainly on laboratory-based studies from developed countries, often with no description of the patient population studied $[5,7,23]$. If smears and cultures were performed and read exactly the same way on specimens from clinically similar patients in a low then in high TB prevalence area, we should observe similar sensitivity. However, because of the higher prevalence of positive smear and culture results observed in high endemic areas, the absolute and relative benefit will also increase, while the NND decreases. Paradoxically, countries with the lowest rates of TB typically do both microscopy and culture, while countries with the highest rates of TB often forego culture due to costs and lack of needed infrastructure. Our data supports recent statements by the $\mathrm{WHO}[9,24-27]$ and the International Standards for TB Care [28] on the use of culture in low income settings, recognizing that for each setting the incremental yield will need to be assessed against the incremental cost of culture-based testing.

In our study, almost $90 \%$ of positive cultures were positive on the first specimen, and the IY of the second culture was $10 \%$. A lower yield (77.6\%) was observed for the first specimen in smear negative SE compared to smear positive SE. These findings were also reported in studies that performed 3 cultures on patients [23,29-31]. However, finding $77 \%$ of culture positive patients with the first specimen is still significant and much more informative than a negative smear result. Table 5 compares our results to some other studies that have performed cultures in TB suspects and where results for first and second specimens were reported. The RY of $1 \mathrm{C}$ vs $1 \mathrm{~S}$ was higher (2.7) in a study performed in HIV patients in Thailand and Vietnam [32], most likely because of the lower yield of light microscopy (Ziehl-Nielsen) compared to fluorescent microscopy.

There were several limitations to our study. First, our analysis was done on $68.9 \%$ of the $3742 \mathrm{~TB}$ suspects with at least 1 smear and 1 culture result. This shows the challenges of obtaining 2 samples per TB suspect in regular programme settings. However, we were able to estimate the proportion of positives for the missing specimens using established formulas [19]. We excluded $15.4 \%$ of SE with contaminated culture results from our analysis in order to calculate the yield of first and second specimens accurately . Lower or similar contamination rates have been reported with the MGIT ${ }^{\circledR}$ system: 8.6\% [33], 13\% [32], 16.9\% [34], 16.4\% [35]. Despite excluding these contaminated results, we were nevertheless able to analyse a

Table 6 Definitions and formulas used for yield calculations

\begin{tabular}{|c|c|c|}
\hline & Description & Formula \\
\hline \multirow[t]{2}{*}{ Ad } & Number of SE who had specimens examined & $P x+N P+N N+N 9$ \\
\hline & Observed \# of P SE after 1 specimen & Px \\
\hline Od & Observed \# of P SE after 2 specimens & $P x+N P$ \\
\hline Sd1 & Fraction of SE found positive on the $1^{\text {st }}$ specimen & $P x /(P x+N P+N N+N 9)$ \\
\hline $\mathrm{Sd} 2$ & Fraction of SE found to be negative on the 1st specimen but positive on the 2 nd & $N P /(N P+N N+N 9)$ \\
\hline \multirow[t]{2}{*}{ Md } & \# of P SE missed by failing to do a 2nd specimen & $\mathrm{Sd} 2 * \mathrm{~N} 9$ \\
\hline & Expected \# of P SE after 1 specimen: same as Observed \# of P SE after 1 specimen & \\
\hline \multirow[t]{2}{*}{ Ed } & Expected \# of P SE after 2 specimens (if all suspects had 2 specimens done) & $\mathrm{Od}+\mathrm{Md}$ \\
\hline & Expected proportion of P SE after 1 specimen: same as Sd1 & \\
\hline $\mathrm{Rd}$ & Expected proportion of P SE after 2 specimens & $\mathrm{Ed} / \mathrm{Ad}$ \\
\hline Fd1 & Fraction for the potential IY from the $1^{\text {st }}$ specimen & $\mathrm{Px} / \mathrm{Ed}$ \\
\hline $\mathrm{Fd} 2$ & Fraction for the potential IY from the $2^{\text {nd }}$ specimen & $(M d+N P x) / E d$ \\
\hline OFP1 & Overall fraction of $\mathrm{P}$ on $1^{\text {st }}$ specimen & $\mathrm{Rd} * \mathrm{Fd} 1$ \\
\hline OFP2 & Overall fraction of $P$ on $2^{\text {nd }}$ specimen & $\mathrm{Rd} * \mathrm{Fd} 2$ \\
\hline NND1 & NND on 1st specimen: number of specimens needed to be examined to find one additional case & 1/OFP1 \\
\hline NND2 & NND on 2nd specimen & 1/OFP2 \\
\hline
\end{tabular}

Appendix Legend. \# = Number, $\mathrm{P}=$ positive, $\mathrm{SE}=$ Suspect Episodes, NND = number needed to diagnose. 
large number of SE. However, in real life, these results have an impact on costs and patient management [34]. Second, the HIV status was available only for treated patients that had consented to HIV testing, or $13.6 \%$ of 2537 SE. HIV voluntary counseling and testing (VCT) was offered at the clinic but low rates could be explained by the absence of routinely available antiretroviral therapy at the time of the study. Third, we were not able to compare the yield of the solid vs liquid culture media, as the individual results for these were not available. However, other studies have shown the higher yield of liquid vs solid culture [32-34]. Finally, some inconsistency rates in smear results were observed. When using all smear positive SE as a denominator, the proportion of SE positive on the first smear was $74.3 \%$, lower than the $85 \%$ reported in a meta-analysis [8]. This may be due to the setting of the study in a regular programme. However, the incremental yield of culture over microscopy in this study is not due to false-positive cultures due to laboratory cross-contamination. We have assessed this possibility in another manuscript, using dummy samples, and found that the specificity of culture in this setting is $98.9 \%$, which is comparable to other rates described in the literature [13].

Recently, a novel and simple nucleic-acid amplification test $\left(\right.$ Xpert $^{\circledR}$ MTB/RIF) has been developed [36] and endorsed by WHO for global implementation [37]. Performing 1 Ziehl-Neelsen smear, 1 Xpert $^{\circledR}$ MTB/RIF and 1 liquid culture on TB suspects yielded $38.4 \%, 46.2 \%$ and $50.1 \%$ positive results respectively. Compared to doing 1 smear, the RY, AY and NND would be $1.2,7.8 \%$ and 13 for the Xpert $^{\circledR}$ MTB/RIF and 1.3, $11.7 \%$ and 9 for culture [36]. However, as stated by WHO, the Xpert MTB/RIF technology does not eliminate the need for conventional microscopy culture and drug susceptibility testing [37]. The rapidity and simplicity of the method are clear advantages. However, sensitivity in smear negative patients remains an issue, with some reported sensitivities of $43.4 \%$ [38], 54.5\% [39] and 72.5\% [36]. Furthermore, drug susceptibility testing is still required to detect resistance to drugs other than rifampicin [37]. As the Xpert technique is implemented, initial studies show it can potentially be cost-effective compared to sputum smear diagnosis and clinical diagnosis in smear-negative cases [40]. Future studies could evaluate the cost-effectiveness of smear versus Xpert, as compared with smear versus culture, or even the integration of two of these tests. Depending on the prevalence of positive samples by new diagnostic techniques, the NND can be determined for either test, in order to predict the incremental gain in case detection, and ultimately, the impact on patient care.

\section{Conclusions}

In conclusion, in a high TB burden setting, the use of culture methods can almost double the number of microbiologically-confirmed cases of TB, with just under 8 cultures required to detect 1 additional case.

\section{Appendix}

Yield calculations (adapted from Rieder et al. [19]).

There are 6 possible patterns among those who had a diagnostic examination (Ad) in a setting where 3 specimens are required: Px, NPx, NNP, NN, NN9 and N99, where $\mathrm{P}$ is a positive smear, $\mathrm{x}$ a subsequent result of no interest, $\mathrm{N}$ a negative result, and 9 a missing result. For our study, since we aimed to collect 2 specimens and since less than $10 \%$ of SE had $\geq 3$ smears or 3 cultures done, we kept 4 patterns ( $\mathrm{Px}, \mathrm{NP}, \mathrm{NN}$ and N9) and adapted the formulas accordingly.

To calculate the incremental yields, the assumption was made that those with an N9 result had the same probability of being positive on the second smear as those with an NP result. Those (positive) missed by failing to do a second smear examination will be denoted as Md (Table 6).

\section{Competing interests}

All authors: no competing interests.

\section{Authors' contributions}

$A M D$ and DC participated in all aspects from study design, data collection, analysis and writing. $A B$ and $M A B$ contributed to statistical analysis and writing. RW, SV, PvH, MAB contributed to study design, data analysis and writing. All authors read and approved the final manuscript.

\section{Acknowledgements}

Work was done through the support of a grant from the Sequella Global Tuberculosis Foundation. AMD was the recipient of fellowship support from the Fonds de la Recherche en Santé du Québec, Canada. We thank Marlein Bosman and Tania Dolby for their work at NHLS, as well as the staff from the Gugulethu TB clinic. The authors dedicate this effort to the memory of Lafras Steyn, who contributed from the inception to the Gugulethu study site.

\section{Author details}

${ }^{1}$ Département de Microbiologie et Immunologie, Université de Montréal, Montreal, QC, Canada. ${ }^{2} \mathrm{CHU}$ Sainte-Justine, 3175 Chemin de la Côte Sainte-Catherine, Montréal, QC H3T 1C5, Canada. ${ }^{3}$ KNCV Tuberculosis Foundation, The Hague, The Netherlands. ${ }^{4} \mathrm{CINIMA}$, Academic Medical Centre Amsterdam, Amsterdam, The Netherlands. ${ }^{5}$ Centre for Infectious Disease Epidemiology and Research, School of Public Health and Family Medicine, University of Cape Town, Cape Town, South Africa. ${ }^{6}$ DST/NRF Centre of Excellence for Biomedical TB Research/ US/MRC Centre for Molecular and Cellular Biology, Division of Molecular Biology and Human Genetics, Department of Biomedical Sciences, Faculty of Health Sciences Stellenbosch University, PO Box 19063, Tygerberg 7505, South Africa. ${ }^{7}$ Department of Medicine, McGill University, Montreal, Québec, Canada. ${ }^{8}$ McGill University Health Centre, Room A5.156, 1650 Cedar Avenue, Montreal, QC H3G 1A4, Canada.

Received: 12 January 2012 Accepted: 5 September 2012 Published: 14 September 2012

\section{References}

1. World Health Organization: An expanded DOTS framework for effective tuberculosis control. WHO/CDS/TB/2002.297. Geneva: WHO; 2002.

2. Enarson DA: International Union Against Tuberculosis and Lung Disease: Management of tuberculosis: a guide for low income countries. 5th edition. Paris: International Union Against Tuberculosis and Lung Disease; 2000.

3. World Health Organization: Laboratory services in tuberculosis control. In Part I: Organization and management. Geneva: WHO; 1998. WHO/TB/98.258. 
4. Pfyffer GE, Palicova F: Mycobacterium: General Characteristics, Laboratory Detection, and Staining Procedures. In Manual of Clinical Microbiology. 10th edition. Edited by Murray PR, Baron EJ, American Society for Microbiology. Washington, DC: ASM Press; 2011:472-502.

5. Lipsky BA, Gates J, Tenover FC, Plorde JJ: Factors affecting the clinical value of microscopy for acid-fast bacilli. Rev Infect Dis 1984, 6:214-222.

6. Van Deun A, Portaels F: Limitations and requirements for quality control of sputum smear microscopy for acid-fast bacilli. Int J Tuberc Lung Dis 1998, 2:756-765

7. Van Deun A: What is the role of mycobacterial culture in diagnosis and case finding? In Toman's tuberculosis. Case detection, treatment and monitoring. 2nd edition. Geneva: World Health Organization; 2004:35-43.

8. Mase SR, Ramsay A, Ng V, Henry M, Hopewell PC, Cunningham J, et al: Yield of serial sputum specimen examinations in the diagnosis of pulmonary tuberculosis: a systematic review. Int J Tuberc Lung Dis 2007, 11:485-495.

9. World Health Organization: Use Of Liquid Tb Culture And Drug Susceptibility Testing (DST). In Low And Medium Income Settings; 2007. http://www.who.int/tb/dots/laboratory/policy/en/index3.html.

10. Aït-Khaled N, Alarcon E, Armengol R, Bissell K, Boillot F, Caminero JA, et al: Management of Tuberculosis: A Guide to the Essentials of Good Clinical Practice. Paris, France: International Union Against Tuberculosis and Lung Disease; 2010.

11. Department of Health Western Cape: HIV Prevalence in the Western Cape Results of the 2006 HIV Antenatal Provincial and Area Surveys. Cape Town, South Africa: Provincial Government of the Western Cape; 2007.

12. World Health Organization: Global tuberculosis control: WHO report 2011. WHO/HTM/TB/2011. 16th edition. Geneva: World Health Organization; 2011. WHO/HTM/TB/2011.16

13. Demers AM, Boulle A, Warren $R$, Verver $S$, van Helden P, Behr MA, et al: Use of simulated sputum specimens to estimate the specificity of laboratorydiagnosed tuberculosis. Int J Tuberc Lung Dis 2010, 14:1016-1023.

14. South African Department of Health: The South African Tuberculosis Control Programme Practical Guidelines. South Africa: Pretoria, South Africa, Department of Health; 2000. 1-47. 2000.

15. World Health Organization: Laboratory services in tuberculosis control. Part II: Microscopy. Geneva: WHO; 1998:1-65. WHO/TB/98.258.

16. Van Deun A, Salim AH, Cooreman E, Daru P, Das AP, Aung KJ, et al: Scanty AFB smears: what's in a name? Int J Tuberc Lung Dis 2004, 8:816-823.

17. Lawson L, Yassin MA, Ramsay A, Emenyonu NE, Squire SB, Cuevas LE: Comparison of scanty AFB smears against culture in an area with high HIV prevalence. Int J Tuberc Lung Dis 2005, 9:933-935.

18. World Health Organization: Same-day-diagnosis of tuberculosis by microscopy - Policy Statement. Geneva: WHO; 2011. WHO/HTM/TB/2011.7.

19. Rieder HL, Chiang CY, Rusen ID: A method to determine the utility of the third diagnostic and the second follow-up sputum smear examinations to diagnose tuberculosis cases and failures. Int J Tuberc Lung Dis 2005, 9:384-391.

20. Bandolier: How good is the test; 1996. http://www.medicine.ox.ac.uk/ bandolier/band27/b27-2.html.

21. Linn S, Grunau PD: New patient-oriented summary measure of net total gain in certainty for dichotomous diagnostic tests. Epidemiol Perspect Innov 2006, 3:11

22. Tuberculosis Division of the International Union Against Tuberculosis and Lung Disease: Tuberculosis bacteriology-priorities and indications in high prevalence countries: position of the technical staff of the Tuberculosis Division of the International Union Against Tuberculosis and Lung Disease. Int J Tuberc Lung Dis 2005, 9:355-361.

23. Urbanczik R: Present position of microscopy and of culture in diagnostic mycobacteriology. Zentralbl Bakteriol Mikrobiol Hyg [A] 1985, 260:81-87.

24. Stop TB, Partnership and World Health Organization: Global Plan to Stop TB 2006-2015. Geneva: WHO; 2006. WHO/HTM/STB/2006.35.

25. World Health Organization: Improving the diagnosis and treatment of smear-negative pulmonary and extra-pulmonary tuberculosis among adults and adolescents. In Recommendations for HIV-prevalent and resourceconstrained settings. Geneva: WHO; 2007. WHO/HTM/TB/2007.379.

26. World Health Organization: Treatment of tuberculosis: guidelines. 4th edition. Geneva: WHO; 2009. WHO/HTM/TB/2009.420.

27. World Health Organization: Framework for Implementing New Tuberculosis Diagnostics; 2010. http://www.who.int/tb/laboratory/ whopolicyframework_rev_june2011.pdf.
28. Tuberculosis Coalition for Technical Assistance: International Standards for Tuberculosis Care (ISTC). 2nd edition. The Hague: Tuberculosis Coalition for Technical Assistance; 2009.

29. Toman K, Frieden T, Toman K, World Health Organization: Toman's tuberculosis. Case detection, treatment, and monitoring: questions and answers. 2nd edition. Geneva: World Health Organization; 2004.

30. Nelson SM, Deike MA, Cartwright CP: Value of examining multiple sputum specimens in the diagnosis of pulmonary tuberculosis. J Clin Microbiol 1998, 36:467-469.

31. Cascina A, Fietta A, Casali L: Is a Large Number of Sputum Specimens Necessary for the Bacteriological Diagnosis of Tuberculosis? J Clin Microbiol 2000, 38:466.

32. Monkongdee $\mathrm{P}, \mathrm{McC}$ arthy KD, Cain KP, Tasaneeyapan T, Nguyen HD, Nguyen TN, et al: Yield of acid-fast smear and mycobacterial culture for tuberculosis diagnosis in people with human immunodeficiency virus. Am J Respir Crit Care Med 2009, 180:903-908.

33. Cruciani M, Scarparo C, Malena M, Bosco O, Serpelloni G, Mengoli C: MetaAnalysis of BACTEC MGIT 960 and BACTEC 460 TB, with or without Solid Media, for Detection of Mycobacteria. J Clin Microbiol 2004, 42:2321-2325.

34. Chihota VN, Grant AD, Fielding K, Ndibongo B, van Zyl A, Muirhead D, et al: Liquid vs. solid culture for tuberculosis: performance and cost in a resource-constrained setting. Int J Tuberc Lung Dis 2010, 14:1024-1031.

35. Ssengooba W, Kiwanuka N, Kateete DP, Katamba A, Joloba ML: Incremental Yield of Serial Sputum Cultures for Diagnosis of Tuberculosis among HIV Infected Smear Negative Pulmonary TB Suspects in Kampala, Uganda. PLoS One 2012, 7:e37650.

36. Boehme CC, Nabeta P, Hillemann D, Nicol MP, Shenai S, Krapp F, et al: Rapid molecular detection of tuberculosis and rifampin resistance. N Engl J Med 2010, 363:1005-1015.

37. World Health Organization: Policy statement: automated real-time nucleic acid amplification technology for rapid and simultaneous detection of tuberculosis and rifampicin resistance: Xpert MTB/RIF system. Geneva: World Health Organization; 2011. WHO/HTM/TB/2011.4.

38. Lawn SD, Brooks SV, Kranzer K, Nicol MP, Whitelaw A, Vogt M, et al: Screening for HIV-associated tuberculosis and rifampicin resistance before antiretroviral therapy using the Xpert MTB/RIF assay: a prospective study. PLoS Med 2011, 8:e1001067.

39. Theron G, Peter J, van Zyl-Smit R, Mishra H, Streicher E, Murray S, et al: Evaluation of the Xpert MTB/RIF assay for the diagnosis of pulmonary tuberculosis in a high HIV prevalence setting. Am J Respir Crit Care Med 2011, 184:132-140.

40. Vassall A, van Kampen S, Sohn H, Michael JS, John KR, DEN BS, et al: Rapid diagnosis of tuberculosis with the Xpert MTB/RIF assay in high burden countries: a cost-effectiveness analysis. PLOS Med 2011, 8:e1001120.

\section{doi:10.1186/1471-2334-12-218}

Cite this article as: Demers et al:: High yield of culture-based diagnosis in a TB-endemic setting. BMC Infectious Diseases 2012 12:218.

\section{Submit your next manuscript to BioMed Central and take full advantage of:}

- Convenient online submission

- Thorough peer review

- No space constraints or color figure charges

- Immediate publication on acceptance

- Inclusion in PubMed, CAS, Scopus and Google Scholar

- Research which is freely available for redistribution 